\title{
EVALUACIÓN DE LA CALIDAD DE LOS SERVICIOS ADMINISTRATIVOS COMO APOYO A LA ACADEMIA EN LA ESCUELA DE QUÍMICA DE LA UNIVERSIDAD DE COSTA RICA
}

\author{
JAVIER QUESADA ESPINOZA \\ Universidad de Costa Rica, Costa Rica \\ francisco.quesada@ucr.ac.cr
}

\section{RESUMEN}

El presente artículo tiene por objeto valorar la satisfacción del estudiantado respecto a la calidad del servicio administrativo brindado en la Escuela de Química de la Universidad de Costa Rica. Se empleó una adaptación del cuestionario del modelo SERVQUAL. Este fue aplicado a 102 estudiantes de la carrera. Se identificó una brecha importante entre la percepción del estudiantado sobre la calidad del servicio y la expectativa que se tenía de él. Todas las dimensiones del modelo presentaron una brecha similar, con una media global para el instrumento de -1.01 ( $\mathrm{s}=$ 0.31). Las dimensiones fueron correlacionadas con características sociodemográficas y se encontraron asociaciones con la edad y avance en la carrera del estudiantado. Se generaron modelos de regresión donde se destaca el avance mencionado como el predictor de la satisfacción. Los resultados muestran que existen puntos de mejora para brindar en servicio administrativo idóneo que apoye a la formación académica.

PALABRAS CLAVE: SERVICIO, CALIDAD, EDUCACIÓN, UNIVERSIDAD, SATISFACCIÓN.
ABSTRACT

The purpose of this paper is to assess student satisfaction with the quality of the administrative service provided at the School of Chemistry of the University of Costa Rica. An adaptation of the SERVQUAL model questionnaire was used. This questionnaire was administered to 102 students at all levels of the Chemistry major. The results revealed an important gap between students' perception of the quality of the service and what it was expected from it. All dimensions of the model presented a similar gap, with a global mean for the instrument of -1.01 ( $s=0.31$ ). The dimensions were correlated with sociodemographic characteristics, which established a relationship between students' age and their progress in the major. Regression models were generated, which highlight students' progress in their major as the predictor of their satisfaction. The results show aspects that need to be improved to provide a suitable administrative service that supports academic training.

KEYWORDS: SERVICE, QUALITY, EDUCATION, UNIVERSITY, SATISFACTION. 


\section{INTRODUCCIÓN}

La calidad del servicio que presta un centro educativo se manifiesta en la conformidad y satisfacción que exprese la comunidad académica (estudiantes, personal administrativo y docente). Estos son de muy diferente índole y son proveídos por actores diversos a clientes igualmente dispares.

Dentro de la estructura contemporánea de la universidad, existen tres ejes primordiales: docencia, investigación y extensión (entendida como vínculo con la sociedad). En cada uno de estos escenarios los participantes pueden variar o cumplir roles diferentes. Si se parte de la premisa de que la docencia es la función central de las universidades, se debe considerar a los estudiantes como los consumidores del servicio que brinda la organización.

Es frecuente escuchar que el estudiantado no es el único cliente de las universidades y que actores como las familias, empresas, gobierno o sociedad en general también se constituyen en clientes. Cada vez más, las instituciones son conscientes de que los estudiantes son el grupo a satisfacer prioritariamente.

En muchas ocasiones, se ha considerado la calidad en la educación como la satisfacción de las necesidades de formación de los alumnos. En este sentido, entran en el panorama, además de los actores antes mencionados, los egresados y los empleadores. Estos clientes se pueden visualizar dentro de una estructura como la que representa el Sistema Nacional de Acreditación de la Educación Superior de Costa Rica (SINAES). Este modelo hace una valoración de los elementos que inciden en la formación adecuada de los estudiantes e incluye muchos aspectos.

Sin embargo, el cliente directo solo conforma una parte en la evaluación y la interpretación de los resultados no se hace únicamente desde la perspectiva del estudiante. Es preciso hacer una lectura más clara de lo que implica el servicio que recibe el estudiantado. Es difícil hacer una segregación de los diferentes servicios que emplean estos, todos se encuentran entrelazados y aportan a la satisfacción global. No obstante, existen dos grandes grupos en donde se pueden categorizar los servicios: el académico y el administrativo.

La mayoría del estudiantado reconoce y valora la calidad del servicio que se brinda por parte de los docentes. En algunos casos, existen rúbricas de evaluación para cuantificar la satisfacción al respecto; pero, en el tema de los servicios administrativos, la valoración no es tan común. Generalmente, esta suele ser una mezcla de los servicios académicos y administrativos. Sin embargo, si el estudiante paga por el servicio logra hacer la diferencia más fácilmente.

La lucha en el mercado de la educación superior es evidente y más notoria en las universidades privadas, donde el servicio brindado debe ser satisfactorio en relación con el pago efectuado. La atracción de nuevos estudiantes es un desafío diario que implica una estrategia de inversión para este fin.

No obstante, este no es un tema que se deba confinar a la educación superior privada. En la educación superior costarricense, las universidades públicas afrontan un gran reto en relación con la capacidad de atraer al estudiantado por parte de las universidades privadas y entre ellas. Los estudiantes del nuevo siglo son más exigentes en las características del centro educativo donde desean llevar a cabo sus estudios y de las condiciones del servicio que estos deben de tener.

A pesar de que el tema ya tiene varios años en el ambiente educativo superior, en la revisión de la literatura no se encontró referencia a estudios en esta línea de investigación en Costa Rica. Como se mencionó anteriormente, la atención se ha enfocado en la acreditación de la calidad de las carreras, pero no se ha profundizado en factores conexos a la enseñanza que son importantes para la 
formación de los estudiantes. Sin embargo, se ha valorado la satisfacción del servicio por diferentes autores alrededor del mundo, con acercamientos muy diferentes e igualmente, con resultados muy heterogéneos.

En este sentido, Hanssen y Solvoll (2015) estudiaron el tema en una universidad de Noruega, midiendo la percepción del estudiantado pero evaluando la satisfacción con las instalaciones donde se brinda el servicio, que solo representa uno de los elementos que otros emplean para definir una calidad del servicio. Identificaron que la calidad de los auditorios, áreas sociales y bibliotecas resultó ser lo que más influenciaba la satisfacción. En la Universidad de Amirkabir, en Irán, los investigadores encontraron que los estudiantes no eran atendidos por personas con el conocimiento del tema que requerían (Abili, Narenji Thani, y Afarinandehbin, 2012).

Lupo (2013) valoró la calidad de la educación superior en Italia; midió una satisfacción global que incluye el servicio académico y administrativo. Algo similar se efectuó en México por dos grupos de investigación diferentes (Álvarez Botello, Chaparro Salinas, y Reyes Pérez, 2014; Candelas Zamorano, Gurruchaga Rodríguez, Mejías Acosta, y Flores Ávila, 2013). En Argentina, Tumino y Poitevin (2013) también lo realizaron, pero enfocándose en detectar posibles diferencias entre los grupos de estudiantes por diversas características sociodemográficas.

Asimismo, en Reino Unido se evaluó la percepción del estudiante sobre una mezcla de factores administrativos y académicos (Oldfield y Baron, 2000). En Indonesia también se ha realizado investigación para establecer la satisfacción del estudiantado. Estos autores aportan una diferenciación por nivel de avance en la carrera, un factor importante de considerar (Sumaedi, Mahatma Yuda Bakti, y Metasari, 2012).

Ur-Rashid y Rhman (2017) encontraron, en Bangladesh, resultados similares a los anteriores con brechas de insatisfacción importantes en la dimensión no académica. Lo mismo se observa en Rusia, en donde un estudio muestra una calidad del servicio baja (Galeeva, 2016). Cheruiyot (2013) evalúa la percepción en la universidad más importante de cada uno de tres países africanos, donde los aspectos relativos a los servicios administrativos son los valorados con más baja puntuación.

En España se desarrolló una investigación en varias sedes de una universidad, donde no se observaron diferencias significativas entre los escenarios mencionados pero sí espacio para mejorar la satisfacción de los estudiantes (Gallifa y Batallé, 2010). En Brasil se realizó un estudio que valoraba expectativas y percepciones. Se observó una variación en la brecha en cada punto de medición, pero no existió diferencia significativa (Campos, Santos, y Castro, 2017).

Muchas de las investigaciones reportadas en la literatura evalúan la calidad de los servicios sin hacer discriminación entre lo académico y administrativo. Sin embargo, existen estudios con solo el segundo enfoque. Por ejemplo, en Irán se encontró insatisfacción (Rezaei et al., 2017). Sharif y Kassim (2012) no solo emplearon la brecha entre percepción y expectativa como manera para analizar la satisfacción de los estudiantes; realizaron análisis multivariado para encontrar relaciones entre las dimensiones.

También se ha investigado cómo repercute la satisfacción de los estudiantes en la intensión de estos en cambiar de universidad. Los servicios administrativos explicaron el $4.1 \%$ de esta intensión. Los resultados tienen mayor relevancia cuando se habla de instituciones de educación superior (IES) de carácter privado, en donde una transferencia a otra universidad implica disminución de ingresos (Al-Kilani y Twaissi, 2017).

Si bien es cierto que la calidad del servicio en la educación superior ha generado un interés mayor en el sector privado, las instituciones públicas 
también han mostrado preocupación por el tema. Esto impacta en la calidad de la formación que directamente repercute en al prestigio de la universidad. Algunos estudios se han efectuado en Latinoamérica, como los ya citados en Argentina, Brasil y México.

Las universidades públicas en Costa Rica aún se encuentran en la transición para entender que el cliente primario de su servicio es el estudiante y que, dentro de una sociedad globalizada que pretende asegurar la calidad de productos y servicios, la calidad de la educación no se puede tener como parámetro único de mejora. Es preciso incorporar elementos propios del servicio administrativo.

\section{EL CASO DE LA ESCUELA DE QUÍMICA DE LA UNIVERSIDAD DE COSTA RICA}

La Universidad de Costa Rica es la institución de educación superior más grande en Costa Rica. Junto con otras cuatro universidades, conforma la oferta de educación superior pública. Dentro de la Facultad de Ciencias se encuentra la Escuela de Química. Fundada en 1956, con la categoría de departamento, ofrece la carrera de química desde esa fecha (Chaverri, 2004).

La Escuela de Química se ubica en el campus central de la universidad, en San Pedro de Montes de Oca, y cuenta con su edificio propio desde 1957. Actualmente, esta unidad académica atiende a 445 estudiantes debidamente matriculados, pero también ofrece cursos a otras carreras, que contempla dar servicio a más de 6500 estudiantes (Camacho, 2018).

Un total de 33 personas efectúan las actividades de carácter administrativo (Escuela de Química, 2018). Esto incluye a personal de consejería, mantenimiento, asistentes de laboratorio, personal de oficina y otras actividades de soporte a la docencia e investigación. Todos los colaboradores tienen, en mayor o menor grado, contacto con los estudiantes.
Una característica importante de los servicios que emplea el estudiantado es que algunas de las tareas que son generalizadas para la institución son brindadas por diferentes dependencias. Actividades relacionadas con los procesos de matrícula, informática, becas, transporte, infraestructura, financieros, entre otros, se encuentran centralizadas.

La carrera de química se encuentra acreditada por el SINAES desde el 2012 y fue re-acreditada en el 2018. Dentro de su compromiso de mejora se contemplan algunos aspectos que tienen que ver con los servicios administrativos; pero, como se mencionó anteriormente, no constituyen el fuerte de la evaluación. Ante esta situación, se plantea una serie de objetivos para el presente estudio con el fin de valorar la satisfacción de los estudiantes en este tema.

En este trabajo se valora la calidad del servicio ofrecido por el sector administrativo de la Escuela de Química de la Universidad de Costa Rica mediante el establecimiento de la brecha entre las percepciones y expectativas de los estudiantes, mediante el modelo SERVQUAL. Además, se pretende definir las dimensiones que tienen más impacto en el nivel de satisfacción de los estudiantes y detectar el efecto de las características sociodemográficas de los estudiantes sobre la percepción de la calidad del servicio recibido.

Este caso de estudio tiene como unidad de análisis la calidad del servicio administrativo. Se aborda mediante la revisión del marco conceptual sobre el tema para, posteriormente, aplicar un instrumento para la valoración de la calidad del servicio y finalizar con su respectiva interpretación.

\section{MARCO TEÓRICO}

La satisfacción del servicio al cliente está enmarcada dentro de una filosofía general de la calidad. Los conceptos que se han desarrollado y que han sido adaptados al tema de interés se originan de 
campos muy diversos y dispares. La adecuación para medir la satisfacción del cliente es particular por estar el componente humano inmerso en la prestación del servicio y la evaluación de la calidad está sujeta a parámetros subjetivos de cada cliente. Seguidamente, algunos aspectos teóricos que sustentan el abordaje que se propone.

\section{¿QUÉ ES CALIDAD?}

Cuando se consulta a las personas sobre el concepto de calidad, muchas pueden expresar lo que entienden por esta desde una experiencia personal. Esto es muy normal, porque a pesar de que se emplea frecuentemente el término, pocas personas pueden definir concretamente qué es calidad. En la mayoría de los casos, esta definición se circunscribe al campo de acción o a la percepción respecto a un bien o servicio que se adquiera.

Lo anterior se observa también en lo realizado por los estudiosos en el tema, quienes coinciden en algunos puntos de sus definiciones, pero no han logrado un consenso. En la tabla 1 se detallan los conceptos esbozados por ocho de los más reconocidos expertos en la materia. Como es evidente, las definiciones propuestas presentan coincidencias y algunas diferencias. En términos generales, se enfocan en requerimientos, algunos determinados intrínsecamente por el producto o servicio que se ofrece y en otros casos por las necesidades o expectativas del usuario o cliente final.

En este sentido es que la International Organization for Standardization (ISO), como ente más reconocido en el área de la calidad, la define como el grado en el cual un set de características inherentes al objeto (producto, servicio, proceso, etc.) Ilena los requerimientos de los clientes, de la legislación o de la práctica común (ISO, 2015). El concepto es resultado de un convenio entre las personas interesadas en establecer especificaciones a cierto objeto, por lo que al ser una construcción no puede ser invariante en el tiempo (Tumino y Poitevin, 2013). Lo anterior brinda una noción de la calidad como concepto, pero es preciso detallar la que se estudia en el presente trabajo: la calidad en el servicio.

\section{TABLA 1}

DEFINICIONES DE CALIDAD DE LOS MÁS REPRESENTATIVOS INTELECTUALES DEL TEMA

\begin{tabular}{|c|c|}
\hline Autor & Definición \\
\hline Philip Crosby & Conformidad con requerimientos. \\
\hline Edwards Deming & $\begin{array}{l}\text { Calidad puede estar definida solamente en términos del agente y reside en la traducción de las necesida- } \\
\qquad \text { des futuras del usuario a características evaluables. }\end{array}$ \\
\hline Armand Feigenbaum & $\begin{array}{l}\text { Las características del producto y servicio provenientes de mercadeo, ingeniería, manufactura y manteni- } \\
\text { miento que estén relacionadas directamente con las necesidades del cliente. }\end{array}$ \\
\hline Kaoru Ishikawa & Calidad significa calidad del producto. Un producto diseñado considerando los requerimientos del cliente. \\
\hline Joseph Juran & $\begin{array}{l}\text { La calidad consiste en aquellas características de producto que se basan en las necesidades del cliente y } \\
\qquad \text { que por eso brindan satisfacción del producto. }\end{array}$ \\
\hline Robert Pirsig & $\begin{array}{l}\text { Define la calidad como excelencia. Significa que se entiende antes de la definición, como una experiencia y } \\
\qquad \text { no una abstracción intelectual. }\end{array}$ \\
\hline Walter Shewhart & $\begin{array}{l}\text { La calidad está determinada de manera subjetiva por lo que el cliente quiere y una objetiva por las propie- } \\
\text { dades del producto, independientemente de lo que el cliente quiere. }\end{array}$ \\
\hline Genishi Tagushi & $\begin{array}{l}\text { La calidad es la pérdida que un producto causa a la sociedad después de ser enviado, aparte de las pérdi- } \\
\text { das causadas por su función intrínseca. Es decir, el producto no cumple con el objetivo especificado. }\end{array}$ \\
\hline
\end{tabular}

Fuente: Elaboración propia con base en Duque, 2005; Duque Oliva y Diosa Gómez, 2014; Hoyer y Hoyer, 2001; Hussain y Birol, 2011. 


\section{LA CALIDAD EN LOS SERVICIOS}

La calidad en los servicios tiene que tratarse de manera diferente porque no puede evaluarse y diagnosticarse de la misma forma que cuando se habla de manufactura. Esto motivó el desarrollo de técnicas particulares para abarcar el tema de la calidad en los servicios (Tumino y Poitevin, 2013).

Existen características particulares que diferencian los servicios de los productos de manufactura. Algunos autores hacen referencia a las siguientes:

- La intangibilidad. La mayoría de los servicios son intangibles. No es posible para el cliente tener un criterio previo a haber recibido el servicio (Duque, 2005; Matsumoto Nishizawa, 2014; Parasuraman, Zeithaml, y Berry, 1985; Tumino y Poitevin, 2013).

- La inseparabilidad. La producción y consumo del servicio se dan de manera simultánea; se realizan al mismo tiempo (Duque Oliva y Diosa Gómez, 2014; Parasuraman et al., 1985; Tumino y Poitevin, 2013).

- La heterogeneidad. Los resultados de la prestación del servicio pueden ser diferentes, afectando la calidad y su evaluación (Duque Oliva y Diosa Gómez, 2014; Matsumoto Nishizawa, 2014; Parasuraman et al., 1985; Tumino y Poitevin, 2013).

- Carácter perecedero. El servicio de extingue en el momento de ser consumido por el cliente. No es posible de almacenar o devolver (Duque, 2005; Tumino y Poitevin, 2013).

- También se incluyen el contacto con el cliente y la relación basada en el cliente como caracterizadores (Duque Oliva y Diosa Gómez, 2014).
Dadas las características señaladas, la calidad del servicio está relacionada con una percepción del desempeño. Este es el fundamento de la catalogación del servicio con base en las expectativas del cliente sobre un intangible que no puede ser valorado previamente. Dentro de este contexto, el concepto hace referencia a una calidad percibida que, por tanto, es subjetiva y va más allá de los atributos del producto. Como consecuencia, la calidad percibida del servicio no puede ser tratada de la misma forma que la calidad de bienes tangibles (Duque Oliva y Diosa Gómez, 2014; Eberle, Milan, y Dorion, 2016).

\section{CALIDAD DEL SERVICIO EN LA EDUCACIÓN SUPERIOR}

La investigación en este tema en el sector de la educación superior es nueva si se le compara con la realizada en el sector comercial. Muchos de los modelos desarrollados para este segundo sector han sido adaptados para el primero. Considerando la calidad total, los usuarios deben ser servidos al máximo grado posible; es decir, el servicio debe satisfacer sus requerimientos y necesidades.

Araya-Castillo (2013) señala "que la calidad de servicio es vista por las universidades como una decisión estratégica para satisfacer las necesidades de sus clientes" (p. 2). Cuando el concepto de calidad de servicio es aplicado al entorno educativo, es necesario tener presente que esta tiene una finalidad, que se lleva a cabo por un proceso de enseñanza-aprendizaje, que implica la acción de los docentes y conlleva la evaluación. Senlle Szodo y Gutiérrez Francescon mencionan que "la calidad no es aplicar las normativas internacionales aprobadas por 157 países o incrementar ya sea horas de clase o los exámenes; la calidad es un cambio profundo en la manera de pensar, de programar, de evaluar y de sentir la educación" (citados por Tumino y Poitevin, 2013, p. 67). 
Tradicionalmente, cuando se habla de calidad de servicio en la educación superior, se valora como servicio educativo. Este posee características propias de los servicios como ser esencialmente intangible, heterogéneo, perecedero y existe una simultaneidad entre la producción y el consumo dentro del proceso enseñanza aprendizaje (Duque Oliva y Diosa Gómez, 2014). Esto lleva a caer en el error de considerar solo la calidad denominada educativa o académica, que en el ámbito latinoamericano se asocia a excelencia y se considera como equivalente a poseer estudiantes sobresalientes y académicos destacados (Álvarez Botello et al., 2014).

Sin embargo, la calidad de la educación difiere de la calidad del servicio en la educación superior. Esta última involucra lo que Álvarez Botello y colaboradores (2014) han definido como:

...la búsqueda de mecanismos para servir y satisfacer al máximo grado posible a los usuarios de los servicios educativos, generando pertinencia y contribuyendo al desarrollo humano sostenible, lo anterior mediante hacer más eficientes los procesos de dirección y liderazgo, desarrollo de procesos académicos, desempeño de los equipos de trabajo y comportamiento de los actores individuales que intervienen en el proceso educativo (p. 8).

Lo anterior presenta una visión general de la calidad del servicio con el propósito de satisfacer las necesidades de los usuarios del sistema de educación superior. Se argumenta que esta es vista por las universidades como una decisión estratégica que procura mejorar la competitividad en el mercado, en especial la educación superior privada (Araya-Castillo, 2013). Los clientes de las universidades son muy diversos y tiene diferentes necesidades, por lo que el concepto puede ser más específico con una definición precisa del usuario.

\section{EL CLIENTE/USUARIO DE LA EDUCACIÓN SUPE- RIOR}

Existe una discusión importante para establecer quién es el cliente o usuario de la educación superior y no se ha llegado a un consenso al respecto. Las definiciones de cliente que se manejan incluyen a los estudiantes, personal administrativo, profesores, gobierno, familias, empresas y la sociedad en general (Araya-Castillo, 2013). Como se muestra, es un panorama muy amplio y con necesidades diferentes que satisfacer.

Algunos autores coinciden en considerar a los estudiantes como las principales partes interesadas en el sector de la educación superior; es decir, sus clientes (Candelas Zamorano, et al., 2013; Correia Loureiro y Miranda González, 2012; Galloway, 1998; Jiménez González, Terriquez Carrillo, y Robles Zepeda, 2011; Marzo Navarro, Pedraja Iglesias, y Rivera Torres, 2005; Torres y Araya, 2010; Tumino y Poitevin, 2013).

La satisfacción de los estudiantes se ha convertido en la medida de los servicios de educación superior que más ha llamado la atención a las universidades en la búsqueda de una ventaja competitiva. Las instituciones no solo deben ocuparse de proporcionar los conocimientos y habilidades que la sociedad requiere de los egresados, sino que también en lo que sienten los estudiantes en su experiencia educativa (ArayaCastillo, 2013).

\section{DIFERENCIA ENTRE LA CALIDAD DEL SERVICIO DE LA EDUCACIÓN Y LA CALIDAD DEL SER- VICIO ADMINISTRATIVO EN LA EDUCACIÓN SUPERIOR}

La calidad en la educación superior se valora desde tres perspectivas: la calidad de la educación, entendida como el proceso que se desarrolla en el aula; la calidad de los servicios administrativos que facilitan la ejecución del proceso educativo; y evaluación de la mezcla de los dos anteriores. 
Parte de la investigación se ha concentrado en valorar la percepción de la calidad por parte de los estudiantes como lo señala Tumino y Poitevin:

Ciertos trabajos se han centrado en la valoración de la experiencia global de los estudiantes en la universidad con relación al conjunto de servicios que ofrece no sólo a nivel docente sino que también se incluyen los servicios de apoyo al estudio y los servicios generales tales como los relacionados con el desarrollo deportivo, cultural y convivencial (Tumino y Poitevin, 2013, p. 67).

También se han publicado muchos trabajos donde el centro de la evaluación de la calidad se concentra en la efectividad de enseñanza y de mecanismos para efectuarla (Oldfield y Baron, 2000). Sin embargo, se ha dado importancia a la calidad del servicio administrativo como un factor que las universidades consideran relevante para mantenerse en un mercado tan competitivo como el actual (AlKilani y Twaissi, 2017; Sharif y Kassim, 2012).

Los servicios administrativos son amplios y contemplan facilidades informáticas, manejo de hospedaje, resolución de problemas de inscripción y matrícula, infraestructura, facilidades para hacer deporte, entre otras (Sharif y Kassim, 2012). También incluye aspectos como amabilidad del personal y complejidad de los asuntos administrativos que están en el marco de un proceso de gestión universitaria que asegura un plan estratégico para la consecución de metas en cada uno de los puntos citados (Đonlagić y Fazlić, 2015; Galeeva, 2016).

Para Gallifa y Batallé (2010), la discusión de la calidad en las instituciones de educación superior se ha enfocado de manera excesiva en el componente académico y ha dejado al margen los aspectos no académicos o administrativos de la calidad. La gestión estratégica de la calidad administrativa es una opción viable que debe ser promovida por los administradores académicos y que se separa de la administración académica de la educación.
Correia Loureiro y Miranda González sostienen que las instituciones de educación superior deben analizar la calidad del servicio prestado para lograr una eficiencia y eficacia en un entorno competitivo y deben definir una estrategia para la satisfacción de las necesidades del cliente, mucho de lo cual pasa por lo administrativo (Correia Loureiro y Miranda González, 2012).

\section{MEDICIÓN DE LA CALIDAD EN EL SERVICIO}

El desarrollo de la calidad del servicio ha sido un tópico de gran interés para los administradores de negocios. Inherentemente se ha trabajado en modelos que sirvan para cuantificarla. Esencialmente han sido generados en Europa, Estados Unidos y otros países desarrollados durante los últimos 30 años (Galeeva, 2016). En la tabla 2 se resumen los principales modelos descritos en la literatura.

El modelo más empleado es el SERVQUAL, el cual se utiliza en el presente estudio. El modelo cuenta con cinco dimensiones y se mide la brecha entre el rendimiento y las expectativas que se tienen. Está constituido por 22 ítems que se valoran en una escala Likert de siete puntos (Bayraktaroglu y Atrek, 2010).

Parasuraman y colaboradores (1988), definen las dimensiones valoradas en el SERVQUAL de la siguiente forma:

1. Tangible. Instalaciones físicas, equipo y apariencia del personal.

- 2. Confiabilidad. Capacidad para realizar el servicio prometido de manera confiable y exacta.

- 3. Capacidad de respuesta. Disposición a ayudar al cliente y proveer el servicio solicitado.

4. Seguridad. Conocimiento y cortesía de los empleados y su habilidad para inspirar confianza y credibilidad. 
TABLA 2

MODELOS IMPLEMENTADOS PARA LA MEDICIÓN DE LA CALIDAD DEL SERVIIIO

\begin{tabular}{|c|c|c|}
\hline \multicolumn{2}{|c|}{ Modelo } \\
\hline Modelo de la imagen & $\begin{array}{c}\text { Desarrollado por la escuela nórdica y formulado por Grönroos, plantea que la calidad percibida por los clientes es la } \\
\text { interacción de la calidad técnica (qué se da) y la calidad funcional (cómo se da) y su relación con la imagen corporativa. }\end{array}$ \\
\hline Modelo de SERVQUAL & $\begin{array}{c}\text { De la escuela estadounidense, Parasuraman, Zeithalml y Berry propusieron un instrumento de medición mediante la } \\
\text { evaluación por separado de las expectativas y percepciones de un cliente. Es el más utilizado por los investigadores. }\end{array}$ \\
\hline Modelo de los tres componentes & $\begin{array}{r}\text { El modelo incluye los elementos de: el servicio y sus características, el proceso de envío o entrega del servicio y el } \\
\text { ambiente que rodea el servicio. Fue propuesto por Rust y Oliver. }\end{array}$ \\
\hline Modelo SERVPERF & $\begin{array}{r}\text { Se trata de una variación al SERVQUAL que cambia el enfoque de la evaluación y las preguntas en los instrumentos. La } \\
\text { propuesta fue realizada por Cronin y Taylor. }\end{array}$ \\
\hline Modelo de desempeño evaluado & Propuesto por Teas, el modelo sugiere puntuaciones ponderadas para el empleo del SERVQUAL. \\
\hline
\end{tabular}

Figura 1

DETALLE DE LAS BRECHAS DENTRO DEL MODELO SERVQUAL

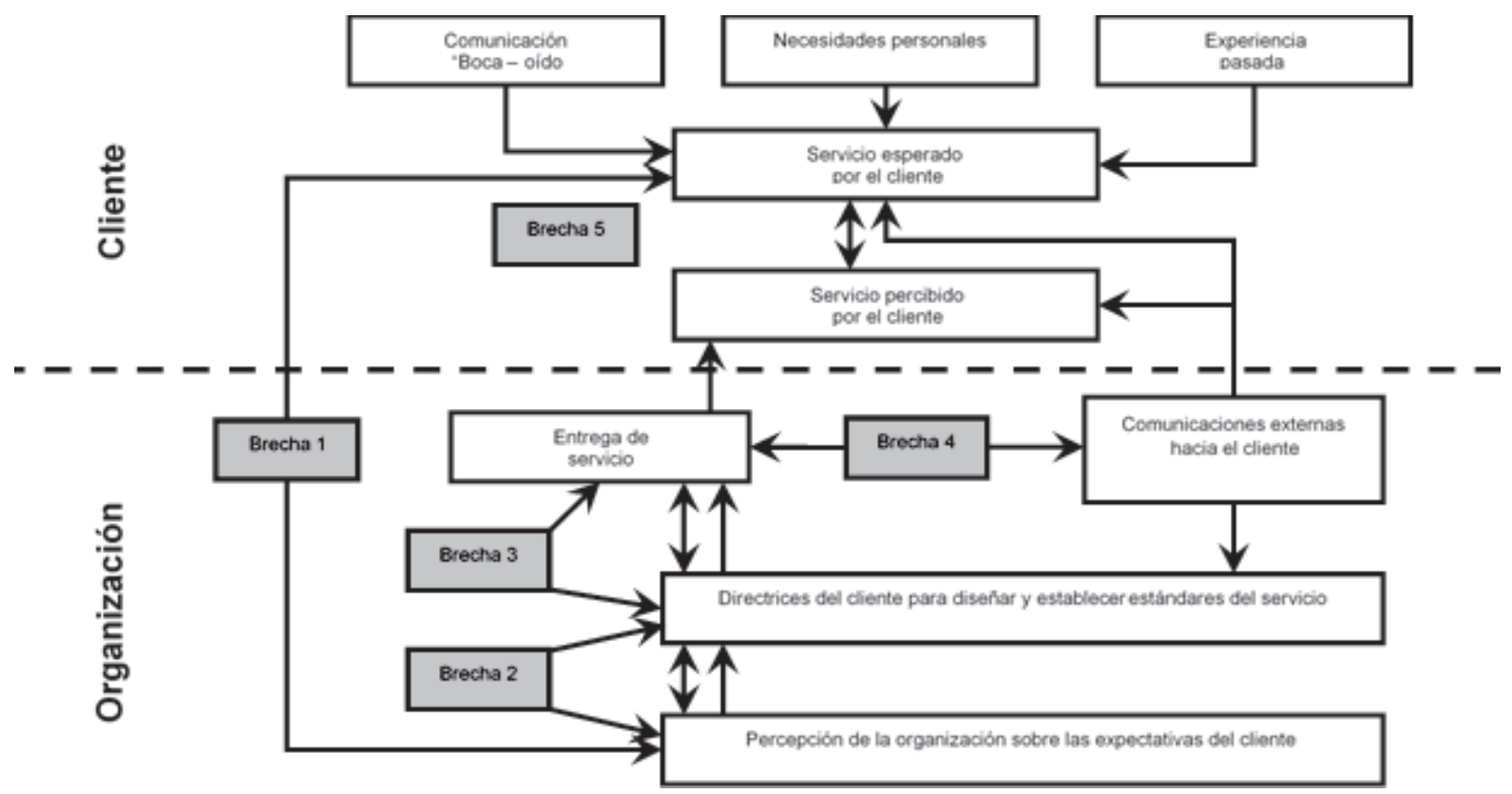

Fuente: Adaptado de Matsumoto Nishizawa, 2014; Parasuraman et al., 1985 
- 5. Empatía. Interés y atención individualizada que la organización provee a sus clientes.

El modelo se basa en las expectativas del cliente, que son creencias sobre el servicio entregado que funcionan como puntos de referencia para valorar el desempeño de la empresa u organización. La expectativa puede variar dependiendo de los estándares previos del cliente. Por otro lado, está la percepción, la cual se refiere a cómo valoran los clientes el servicio ofrecido y que se enlaza con las dimensiones descritas arriba para el SERVQUAL (Matsumoto Nishizawa, 2014; Pereira Puga, 2014; Rezaei et al., 2017).

Los autores del modelo asocian el SERVQUAL al modelo de brechas (denominadas vacíos por algunos autores) que indican las diferencias entre las expectativas y las percepciones, siendo un valor pequeño un indicador de satisfacción. Estas brechas se dividen en cinco. Se explican a continuación y se detalla su interacción es el sistema cliente-empresa en la figura 1 (Matsumoto Nishizawa, 2014, p. 187).

- Brecha 1. Diferencia entre las expectativas de los clientes y las percepciones de los directivos de la organización. Es decir, la capacidad de comprensión de los directivos de las necesidades de sus clientes.

- Brecha 2. Diferencia entre las percepciones de los directivos y las especificaciones de las normas de calidad.

- Brecha 3. Discrepancia entre las especificaciones de la calidad del servicio y la prestación de este.

- Brecha 4. Discrepancia entre la prestación del servicio y la comunicación externa (las promesas que la organización hace).
- Brecha 5. Es la brecha global. Es la diferencia entre las expectativas de los clientes frente a las percepciones de ellos.

El modelo de SERVQUAL es una propuesta general que ha sido aplicada en muchos ámbitos con las adecuaciones necesarias; entre ellos, el de la educación superior privada y pública. Este se enfoca en valorar la calidad global que se encuentra representada por la brecha 5 .

\section{METODOLOGÍA}

En los siguientes apartados se describe la metodología que se siguió para alcanzar los objetivos planteados.

\section{TIPO DE INVESTIGACIÓN, ENFOQUE Y DISEÑO}

Esta investigación es de tipo aplicada, teniendo como caso de estudio la Escuela de Química de la Universidad de Costa Rica. Es un trabajo con un enfoque cuantitativo, multivariado, con un único punto de medición (transversal). Se trata de un estudio no experimental, tipo encuesta y se implementó por medio de dos tipos de análisis (a) análisis descriptivo, y (b) correlaciones y modelos de regresión multivariados (regresión múltiple).

\section{MUESTRA Y DATOS}

La población entrevistada está constituida por los estudiantes de la carrera de química de la Universidad de Costa Rica de todos los niveles del plan de estudios.

\section{PROCEDIMIENTO DEL ESTUDIO Y DE LA RECO- LECCIÓN DE LOS DATOS}

El proyecto incluyó las siguientes etapas:

Etapa 1. La recolección de datos se efectuó en una sola fase, mediante la visita a las aulas de clase. 
Etapa 2. Se realizó el estudio de datos para satisfacer los objetivos planteados por medio de análisis que se detallarán más adelante.

\section{DESCRIPCIÓN DE LOS INSTRUMENTOS PARA LA COLECTA DE DATOS}

Los datos se recolectaron por medio del cuestionario SERVQUAL, adecuado para el servicio administrativo en la educación superior, que valora las percepciones y expectativas de los estudiantes. Consta de 22 ítems con valoración en una escala Likert de 1 a 7 . Además, un cuestionario adicional para caracterizar sociodemográficamente a los encuestados.

\section{DESCRIPCIÓN DE LOS ANÁLISIS UTILIZADOS}

Por los objetivos planteados fue preciso establecer las brechas existentes en cada una de las dimensiones del modelo SERVQUAL. Se emplearon modelos de correlación para establecer el impacto de las dimensiones y un análisis de regresión múltiple para definir el tipo de efecto de las características sociodemográficas.

\section{ANÁLISIS Y DISCUSIÓN DE RESULTA- DOS}

Se aplicaron 102 cuestionarios SERVQUAL adaptado a estudiantes de diferentes niveles de la carrera de Química de la Universidad de Costa Rica. El detalle del género, procedencia y si contaba con beca socioeconómica brindada por la UCR se muestra en la tabla 3. Estos se distribuyen en 10 niveles de avance en la carrera, donde la muestra se concentra en los semestres 5, 7 y 8, con cerca del $60 \%$ de la participación.

\section{CONFIABILIDAD DE LA MEDICIÓN}

La confiabilidad de la medición es un procedimiento que valora hasta qué punto los mismos resultados pueden ser repetidos en pruebas posteriores (Al-Kilani y Twaissi, 2017). Para tales efectos se emplea el coeficiente de alfa de Cronbach, que se calculó para los cuestionarios aplicados tanto en la valoración de expectativas como de percepción. Los valores presentados en la tabla 4 muestran que las diferentes dimensiones en que se subdivide el instrumento de medición tienen coeficientes aceptables, exceptuando la dimensión de tangibles. Según Nunnally (citado por Marzo Navarro et al., 2005) un valor superior a 0.6 se considera aceptable, pero al no alcanzar esta dimensión, este umbral no se consideró en los posteriores análisis. El coeficiente del instrumento completo es muy alto, lo que indica una buena confiabilidad global.

\section{ANÁLISIS DE BRECHA}

Para evaluar la satisfacción sobre el servicio recibido, el primer análisis realizado fue el de brecha. Este consta de una diferencia entre la percepción y la expectativa de la persona que llena el cuestionario. Para cada dimensión de SERVQUAL se calculó la satisfacción y se encontraron las brechas que se muestran en la tabla 5. En ninguna el servicio es considerado como satisfactorio para el estudiantado. La que menos diferencia presenta es la de tangible que, sin embargo, es una dimensión con confiablidad baja.

En esta. el ítem que peor resultado presenta es el referente a la cantidad de equipo para educación e infraestructura $(-1.53, s=1.50)$. No obstante, también incluye el ítem apariencia física del personal que es el que muestra menor brecha de todos los planteados en el cuestionario (-0.04, $s=1.50)$.

Para el caso de la dimensión capacidad de respuesta, la brecha es mayor $(-1.07, s=0.22)$ con valores altos para el ítem de suministro de información adecuada cuando el estudiante la requiere $(-1.21, s=1.37)$. Es un punto de especial atención si se considera que una de las funciones del personal es acompañar al estudiantado en los procesos burocráticos que suelen ser complejos para 
TABLA 3

DETALLE SOCIODEMOGRÁFICO DE LA MUESTRA

\begin{tabular}{|c|c|c|c|}
\hline Característica & Etiqueta & $N$ & Porcentaje \\
\hline \multirow[t]{2}{*}{ Sexo } & Hombre & 47 & 46.1 \\
\hline & Mujer & 55 & 53.9 \\
\hline \multirow[t]{2}{*}{ Procedencia } & Rural & 33 & 32.4 \\
\hline & Urbana & 69 & 67.6 \\
\hline \multirow{2}{*}{$\begin{array}{l}\text { Colegio de proce- } \\
\text { dencia }\end{array}$} & Público & 57 & 55.9 \\
\hline & Privado & 45 & 44.1 \\
\hline \multirow[t]{2}{*}{ Recibe beca } & $\mathrm{Si}$ & 51 & 50.0 \\
\hline & No & 51 & 50.0 \\
\hline
\end{tabular}

Fuente: Elaboración propia, 2018.

TABLA 4

VALORES DE COEFICIENTE ALFA DE CRONBACH COMO MEDIDA DE CONFIABILIDAD

\begin{tabular}{|c|c|c|c|c|}
\hline Dimensión & $\begin{array}{c}\text { Alfa de Cron- } \\
\text { bach }\end{array}$ & Dimensión & Alfa de Cronbach & Número de ítems \\
\hline Expectativa tangibles & .55 & Percepción tangibles & .58 & 4 \\
\hline Expectativa respuesta & .88 & Percepción respuesta & .87 & 5 \\
\hline Expectativa confiabilidad & .75 & Percepción confiabilidad & .83 & 5 \\
\hline Expectativa empatía & .86 & Percepción empatía & .88 & 4 \\
\hline Expectativa seguridad & .82 & Percepción seguridad & .74 & 4 \\
\hline Completo & .93 & Completo & .94 & 22 \\
\hline
\end{tabular}

Fuente: Elaboración propia, 2018.

TABLA 5

BRECHAS REGISTRADAS PARA LAS DIMENSIONES MEDIDAS POR EL SERVQUAL

\begin{tabular}{|c|c|c|}
\hline Dimensión & M & DT \\
\hline Tangibles & -0.85 & 0.61 \\
\hline Capacidad de respuesta & -1.07 & 0.22 \\
\hline Confiabilidad & -0.97 & 0.34 \\
\hline Empatía & -1.17 & 0.11 \\
\hline Seguridad & -1.00 & 0.23 \\
\hline
\end{tabular}

Fuente: Elaboración propia, 2018.

él. La dimensión de confiabilidad mantiene valores similares $(-0.97, s=0.34)$ con un valor muy dispar con respecto a los otros ítems en lo referente a la eficiencia del servicio y la necesidad de darle seguimiento $(-1.55, s=1.64)$.
Incluso este es el ítem con mayor brecha de todos los consultados. Esto muestra que la burocracia no apoya al estudiantado en sus necesidades complementarias en su proceso académico. 
Las últimas dos dimensiones no difieren mucho de las anteriores. La empatía fue la evaluada con el valor más extremo $(-1.17, s=0.11)$, con brechas altas para todos los ítems. Si se habla de servicio al cliente y siendo el estudiantado cliente principal del servicio de la educación superior, que no se tenga empatía con este resulta preocupante y una alerta para revisar el enfoque que tiene la Escuela de Química y la Universidad de Costa Rica en este sentido.

La dimensión de seguridad también mostró una brecha significativa $(-1.00, s=0.22)$ con un ítem sobresaliente de forma negativa: el estudiantado no tiene que esperar mucho tiempo por los servicios que necesitan. Al igual que la dimensión anterior, el sistema no está dando la importancia necesaria a su cliente.

Tomando como base lo anteriormente presentado, no se puede establecer una dimensión que tenga más peso sobre la satisfacción global del estudiantado. La media de esta brecha global es -1.01 ( $s=0.31$ ), valor que se encuentra muy cercano a los valores individuales de las dimensiones.
No se debe dejar de mencionar que las brechas apuntadas son importantes de considerar; sin embargo, en la escala de valoración de 1 a 7 la media de la percepción global de la calidad del servicio es de 4.97 ( $s=0.53$ ) lo cual también indica que la expectativa del estudiantado es bastante alta.

\section{RELACIÓN ENTRE LAS CARACTERÍSTICAS SO- CIODEMOGRÁFICAS Y LA SATISFACCIÓN}

Uno de los objetivos planteados en este estudio fue explorar cómo se relaciona la satisfacción manifestada por los estudiantes y las características sociodemográfica del estudiantado. Se realizó un análisis de asociación de las dimensiones del SERVQUAL y las variables que caracterizan al estudiantado parte de la muestra. Para tal fin, se efectuó una correlación bivariada de Pearson, cuyos resultados se muestran en la tabla 6 .

Las dimensiones de confiabilidad y empatía se asocian negativamente con la edad del estudiante ( $r=-0.319$ y -0.362 respectivamente). La satisfacción en las escalas mencionadas disminuye conforme las personas que llenaron el cuestionario tienen más edad. Un comportamiento semejante

TABLA 6

MATRIZ DE CORRELACIÓN DE PEARSON PARA LAS DIMENSIONES Y VARIABLES SOCIODEMOGRÁFICA

\begin{tabular}{|c|c|c|c|c|}
\hline & Satisfacción respuesta & Satisfacción confiabilidad & Satisfacción empatía & Satisfacción seguridad \\
\hline Frecuencia de uso del servicio & -.173 & -.067 & -.095 & -.047 \\
\hline Edad del estudiante & $-.267^{* *}$ & $-.319^{* *}$ & $-.362^{* *}$ & $-.290^{* *}$ \\
\hline Sexo del estudiante & -.161 & -.133 & -.087 & -.012 \\
\hline Procedencia del estudiante & .059 & -.006 & .017 & .030 \\
\hline Tipo de colegio de procedencia & $-.220^{*}$ & -.190 & -.086 & -.142 \\
\hline Año de ingreso a la universidad & $.308^{* *}$ & $.313^{* *}$ & $.366^{* *}$ & $.277^{* *}$ \\
\hline Cuenta con beca socioeconómica de la UCR & -.135 & -.184 & -.079 & -.133 \\
\hline Semestre que cursa el estudiante & $-.405^{* *}$ & $-.401^{* *}$ & $-.427^{* *}$ & $-.341^{* *}$ \\
\hline
\end{tabular}

${ }^{*} p<.05 ;{ }^{* *} p<.01$

Fuente: Elaboración propia, 2018. 
fue observado por Tumino y Poitevin (2013), para algunas de las dimensiones que evaluaron. Llama la atención que para las otras dimensiones también se nota una correlación inversa. Esta tendencia puede deberse a una mayor exposición al servicio y, por ende, una evaluación con más argumentos valorativos.

También podría deberse a un aumento en las expectativas de cada entrevistado del nivel de servicio que se considera óptimo. Santiñá y colaboradores (citados por Tumino \& Poitevin, 2013) estudiaron el tema y señalaron la "necesidad de realizar estudios de opinión o de evaluación de la calidad de los servicios, por grupos de edad, con el objetivo de adecuar los servicios a las necesidades y expectativas de cada grupo poblacional" (p. XX).

Por otra parte, la correlación entre las dimensiones respuesta $(r=0.308)$, confiabilidad $(r=0.313)$ y empatía ( $r=0.366)$ es positiva con respecto al año de ingreso a la universidad. Esto resulta contradictorio con el comportamiento respecto a la edad, pues este no debería diferir sustancialmente dado que la gran mayoría de estudiantes que ingresan a la universidad lo hacen a una edad similar, por lo que estas dos variables están íntimamente relacionadas.

El semestre que cursa sí presenta una correlación negativa con las dimensiones de respuesta $(r=-0.405)$, confiabilidad ( $r=-0.401)$, empatía $(r=-0.427)$ y seguridad ( $r=-0,341)$. Estos resultados sí coinciden con los descritos para edad, incluso con mayor magnitud. Igualmente se puede esbozar una justificación especulativa, como se realizó anteriormente.

Las variables de frecuencia de uso del servicio, sexo, procedencia del estudiante, tipo de colegio de procedencia y el disfrute de beca no presentan correlación significativa con ninguna de las dimensiones estudiadas. Uno de los presupuestos empleados al definir las variables de estudio fue la diferencia entre los estudiantes procedentes de zona rural y zona urbana. Se esperaba que los provenientes de la segunda zona fueran más susceptibles a valorar la calidad de manera más severa, pero esto fue totalmente refutado por los resultados de coeficiente de Pearson muy cercanos a cero, lo que, en definitiva, muestra que no hay tal influencia.

\section{PREDICCIÓN DE LAS DIMENSIONES DEL SERVI- CIO AL CLIENTE}

Además de evaluar la asociación entre las variables sociodemográficas y las dimensiones del SERVQUAL, explicar cómo las primeras pueden predecir las segundas resulta de interés en el presente trabajo. Por las características de la población sujeta a estudio, se plantearon variables que se presumía estarían relacionadas con la percepción de la calidad, principalmente, aspectos asociados a situaciones socioeconómicos o a procedencia del estudiante. Por este motivo, se incluyó al tipo de colegio y zona de procedencia, así como el disfrute de beca de ayuda socioeconómica. El otro grupo de variables importantes tiene que ver con la edad o antigüedad del estudiante y su contacto con el servicio, aspectos que se consideraba pueden impactar la percepción por el aumento de criterio para poder valorar el servicio recibido.

Las regresiones múltiples efectuadas mostraron que, para el caso de la satisfacción en la dimensión de respuesta, las dos variables predictoras encontradas fueron semestre que cursa el estudiante y frecuencia de uso del servicio. Un 22\% de la variabilidad en la satisfacción está predicha por estas dos variables. Además, los valores de B indican que la satisfacción disminuye para cada una de las variables señaladas. En la tabla 7 se muestra los coeficientes de regresión no estandarizados (B), intercepto, coeficientes de regresión estandarizados ( $\beta$ ), las correlaciones parciales (cambio en R2), R, R2 y R2 ajustado después de introducida cada variable independiente $(\mathrm{VI})$ al modelo.

En el caso de la dimensión de confiabilidad, el 16\% de su variabilidad se debe a la variable semestre que cursa el estudiante. El coeficiente de correlación es de 0.40 y el impacto sobre el cambio de la satisfacción es negativo; es decir, cuanto mayor sea 
TABLA 7

VALORES DE REGRESIÓN SECUENCIAL DE SEMESTRE QUE CURSA EL ESTUDIANTE Y FRECUENCIA DE USO DEL SERVICIO SOBRE LA SATISFACCIÓN DE RESPUESTA

\begin{tabular}{|c|c|c|c|c|}
\hline VI & B & Error estándar de $B$ & $\beta$ & Cambio en $R^{2}$ \\
\hline Semestre que cursa el estudiante & -1.14 & $0.23^{* * *}$ & -0.45 & $0.16^{* * *}$ \\
\hline Frecuencia de uso del servicio & -1.20 & $0.44^{*}$ & -0.25 & $0.06^{* *}$ \\
\hline Intercepto & 5.15 & 2.21 & & \\
\hline $\begin{array}{l}R^{2} \\
R^{2} \text { ajustado } \\
R\end{array}$ & & $\begin{array}{l}0.22 \\
0.21 \\
0.47\end{array}$ & & \\
\hline
\end{tabular}

*** $p<.001 ;{ }^{* *} p<.01$

Fuente: Elaboración propia, 2018.

TABLA 8

VALORES DE REGRESIÓN SECUENCIAL DE SEMESTRE QUE CURSA EL ESTUDIANTE SOBRE LA SATISFACCIÓN DE CONFIABILIDAD

\begin{tabular}{|l|c|c|c|c|}
\multicolumn{1}{|c|}{ VI } & B & Error estándar de $B$ & $\beta$ & Cambio en $R^{2}$ \\
\hline Semestre que cursa el estudiante & -0.70 & $0.16^{* * *}$ & -0.40 & $0.16^{* * *}$ \\
\hline \multicolumn{1}{|c|}{ Intercepto } & -0.26 & 0.96 & & \\
\hline$R^{2}$ & & 0.16 & \\
$R^{2}$ ajustado & & 0.15 & \\
$R$ & & 0.40 & \\
\hline
\end{tabular}

${ }^{* * *} p<.001$

Fuente: Elaboración propia, 2018.

TABLA 9

VALORES DE REGRESIÓN SECUENCIAL DE SEMESTRE QUE CURSA EL ESTUDIANTE SOBRE LA SATISFACCIÓN EMPATÍA

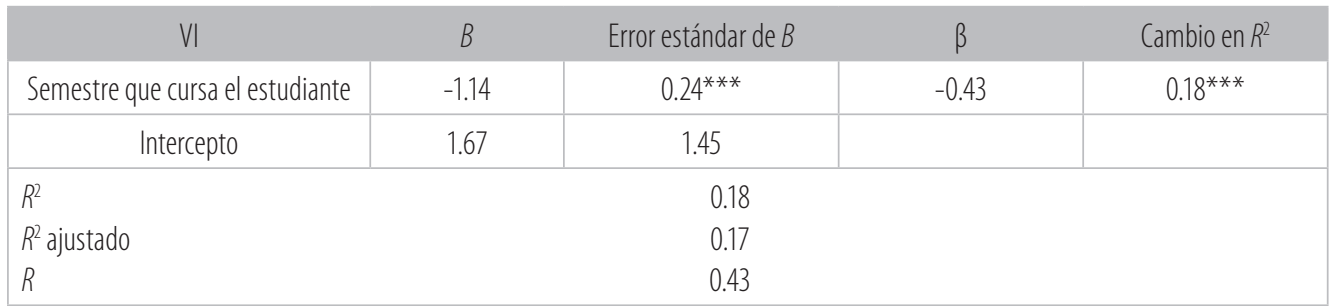

${ }^{* * *} p<.001$

Fuente: Elaboración propia, 2018.

TABLA 10

VALORES DE REGRESIÓN SECUENCIAL DE SEMESTRE QUE CURSA EL ESTUDIANTE SOBRE LA SATISFACCIÓN DE SEGURIDAD

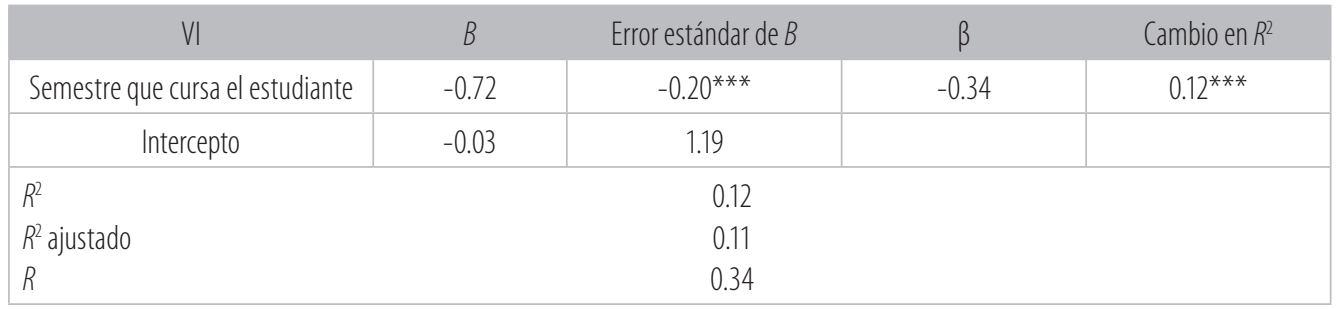

${ }^{* * *} p<.001$

Fuente: Elaboración propia, 2018. 
el semestre en el que se encuentra el estudiante, mayor es la brecha para esta dimensión. El detalle de los datos se presenta en la tabla 8.

La satisfacción para la dimensión empatía presenta el mismo predictor que para el caso anterior. Con un coeficiente de correlación de 0.43 y explicando el 18\% de la variabilidad de la dimensión. Análogamente, se observa que el aumento en el semestre de carrera disminuye la satisfacción como se muestra en la tabla 9.

Finalmente, la dimensión seguridad manifiesta el mismo comportamiento de las dos anteriores, siendo el semestre que cursa el único predictor con un coeficiente de correlación de 0.34 y explicando el $12 \%$ de la variabilidad de esta dimensión. También se observa una asociación negativa entre ambas variables que se detalla en la tabla 10.

Es llamativo el comportamiento reiterado de relación entre el semestre que cursa el estudiante y la satisfacción en las diferentes dimensiones. En particular, que el año de ingreso (que podría estar relacionado directamente con el semestre que cursa) y la edad no sean predictores importantes. La experiencia o madurez no es el punto decisivo en definir si el servicio es bueno o malo, pero sí lo es el hecho de haber experimentado otros servicios en el transcurso de la vida universitaria que solo son empleados con el avance en la carrera o que se tornan complicados con este.

Por otro lado, se observa que el sexo del estudiante, procedencia, tipo de colegio, frecuencia de uso del servicio (excepto para la dimensión respuesta) y tenencia de beca socioeconómica no predicen el comportamiento respecto a la satisfacción en las diferentes dimensiones del SERVQUAL. No tienen ninguna influencia sobre los modelos encontrados, dejando de manifiesto que los antecedentes del estudiantado no son determinantes.

\section{IMPLICACIONES EN LA ADMINISTRACIÓN DE LA ESCUELA DE QUÍMICA}

Las brechas de satisfacción determinadas para cada una de las dimensiones ponen de manifiesto que existe un espacio de mejora importante para la administración universitaria. Si bien es cierto que la magnitud de estas no parece muy grande y que los valores absolutos son satisfactorios dentro de la escala empleada, es necesario señalar que, como característica particular de la sociedad costarricense, se nota una valoración siempre bastante alta al llenar cuestionarios de este tipo. Esto significa que una brecha de una unidad es motivo de llamar la atención porque representa una diferencia sustancial.

Quizás la primera implicación que debería acarrear los resultados mostrados es el establecimiento de un sistema de evaluación de la calidad de servicio de forma sistemática. Este debe contemplar un desglose detallado de los puntos a ser valorados para poder identificar claramente las particularidades a mejorar. El cuestionario aplicado ya brinda algunas aproximaciones generales de qué aspectos considerar como inicio de un proceso de mejora.

Las principales correlaciones o predicciones encontradas se asocian a variables que tienen que ver con el tiempo de estancia del estudiantado en la carrera y ligeramente con la frecuencia de uso del servicio. Esto debe llamar la atención a las autoridades universitarias, pues la calidad del servicio debe mantenerse durante todo el proceso formativo del estudiantado. Es preciso identificar las actividades que deterioran el servicio durante el transcurso de los años de permanencia en la universidad o bien, si se trata de servicios que solo se emplean cuando el estudiantado presenta un avance mayor un su plan de estudios.

\section{CONCLUSIONES}

La satisfacción en el servicio administrativo de la Escuela de Química de la Universidad de Costa 
Rica no se encuentra en un estado crítico; sin embargo, es susceptible a mejoras con base en el análisis de brecha efectuado. No se observa una dimensión que muestre una situación grave; por el contrario, las 5 dimensiones se compartan de manera similar.

Los análisis multivariantes efectuados logran demostrar que existe una correlación entre el grado de satisfacción encontrado y variables ligadas a la antigüedad en la utilización de los servicios. Algo similar ocurre cuando se valora las variables que pueden predecir la satisfacción en las diferentes dimensiones del SERVQUAL, quedando claro que el semestre de la carrera en que se encuentra el o la estudiante explica la mayor variabilidad.

La Escuela de Química debe de tomar en consideración la opinión del estudiantado y comprender la importancia que el soporte administrativo tiene dentro de la gestión académica de la carrera de Química. Debe ir más allá de las exigencias planteadas por un sistema de acreditación de la calidad de la educación y reconocer el impacto de un buen desempeño administrativo en la consecución de los logros académicos de su población estudiantil.

Existen oportunidades de mejora que deben ser atendidas desde las autoridades universitarias para brindar al estudiantado un servicio de calidad que cubra sus necesidades (no deseos) y sirva como un verdadero soporte a la formación académica de los futuros profesionales. Esta primera aproximación muestra algunos de los temas que deben ser atendidos con mayor premura, ya sea en dimensiones o ítems específicos donde las valoraciones fueron menos favorables.

En un sistema administrativo tan complejo como el de la Universidad de Costa Rica, donde algunas actividades se encuentran centralizadas y otras dependen de las unidades académicas, una respuesta rápida puede ser iniciada por estas segundas. Algunas pequeñas mejoras no implican erogaciones importantes, pero sí hacen la diferencia en la satisfacción del cliente.

El sector administrativo debe reconocer su importancia dentro de la estructura de servicio de educación superior que brinda la universidad. Esta identificación debe estar asociada a una mejora en la calidad del servicio admitiendo al estudiantado como cliente principal del proceso.

Son muchos los temas valorados que presentan una brecha importante con respecto al ideal de los y las estudiantes. Este trabajo brinda la guía para el inicio de este proceso de mejora, pero además abre un abanico de posibles estudios que complementen la identificación de falencias, como la valoración que pueda hacer el otro grupo importante de personas que usan los servicios administrativos desde el enfoque académico: el personal docente.

Claro está que falta mucho trabajo por realizar para poder caracterizar bien qué aspectos atender primero y cómo hacerlo, pero también es claro que el estudiantado manifiesta que el servicio actual aún no cumple con su expectativa y por ende es preciso subsanar el problema.

\section{REFERENCIAS}

Abili, K., Narenji Thani, F., y Afarinandehbin, M. (2012). Measuring university service quality by means of SERVQUAL method. Asian Journal on Quality, 13(3), 204-211. doi: 10.1108/15982681211287766

Al-Kilani, M. H. y Twaissi, N. (2017). Perceived quality of administrative services and its consequences on students' behavioral intentions. International Journal of Quality and Service Sciences, 9(1), 103119. doi: 10.1108/IJQSS-09-2016-0064

Álvarez Botello, J., Chaparro Salinas, E. M., y Reyes Pérez, D. E. (2014). Estudio de la satisfacción de los estudiantes con los servicios educativos brindados por instituciones de educación superior del valle de Toluca. Revista Iberoamericana sobre calidad, eficacia y cambio en educación, 13(2), 5-26. 
Araya-Castillo, L. (2013). ¿Qué hemos aprendido sobre la calidad de servicio en educación superior? Revista Pilquen, 16(2), 1-12.

Bayraktaroglu, G. y Atrek, B. (2010). Testing the Superiority and Dimensionality of SERVQUAL vs . SERVPERF in Higher Education. The Quality Management Journal, 17(1), 47-59. Recuperado de http://search.proquest.com.rproxy.uwimona. edu.jm/docview/213591799? accountid $=42530$

Camacho, G. (2018). Comunicación personal. San José.

Campos, D. F., dos Santos, G. S., y Castro, F. N. (2017). Variations in student perceptions of service quality of higher education institutions in Brazil: a longitudinal study. Quality Assurance in Education, 25(4), 394-414. doi: 10.1108/QAE-02-2016-0008

Candelas Zamorano, C. O., Gurruchaga Rodríguez, M. E., Mejías Acosta, A., y Flores Ávila, L. C. (2013). Medición de la satisfacción estudiantil universitaria: un estudio de caso en una institución mexicana. Iberoamerican Journal of Industrial Engineering, 5(9), 261-274.

Chaverri, G. (2004). Historia del desarrollo de la química en Costa Rica (1 era). San José, Costa Rica: Editorial de la Universidad de Costa Rica.

Cheruiyot, T. K. (2013). Service quality and relative performance of public universities in East Africa. The TQM Journal, 25(5), 533-546.

Correia Loureiro, S. M. y Miranda González, F. J. M. (2012). DUAQUAL: Calidad percibida por docentes y alumnos en la gestión universitaria. Cuadernos de Gestion, 12(1), 107-122. doi: 10.5295/ cdg.100251sc

Cronin, J. J. y Taylor, S. A. (1992). Measuring Service Quality: A Reexamination and Extension. Journal of Marketing, 56(3), 55-68. doi: $10.1177 / 002224299205600304$

Đonlagić, S. y Fazlić, S. (2015). Quality Assessment in Higher Education using the Servqual Model. Management, 20(1), 39-57. Recuperado de https:// www.efst.hr/management/Vol20No1-2015/3_ Djonlagic_Fazlic.pdf
Duque, E. (2005). Revisión del concepto de calidad del servicio y sus modelos de medición. Innovar, 15(25), 64-80. http://doi.org/http://moodle2.unid.edu.mx/dts_cursos_mdl/pos/MDL/AC/ AM/AF/Revision.pdf

Duque Oliva, E. J. y Diosa Gómez, Y. (2014). Evolución conceptual de los modelos de medición de la percepción de calidad del servicio: Una mirada desde la educación superior. Suma de Negocios, 5(12), 180-191. doi: 10.1016/S2215910X(14)70040-0

Eberle, L., Milan, G. S., y Dorion, E. (2016). Service quality dimensions and customer satisfaction in a Brazilian university context. Benchmarking: An International Journal, 23(7), 1697-1716. doi: 10.1108/ BIJ-09-2014-0089

Escuela de Química. (2018). Escuela de Química. Recuperado el 26 de marzo, 2018, de http://www. quimica.ucr.ac.cr/administrativo

Galeeva, R. B. (2016). SERVQUAL application and adaptation for educational service quality assessments in Russian higher education. Quality Assurance in Education, 24(3), 329-348. doi: 10.1108/ QAE-06-2015-0024

Gallifa, J. y Batallé, P. (2010). Student perceptions of service quality in a multi-campus higher education system in Spain. Quality Assurance in Education, 18(2), 156-170. doi: 10.1108/09684881011035367

Galloway, L. (1998). Quality perceptions of internal and external customers: A case study in educational administration. TQM Magazine, 10(1), 20-26.

Hanssen, T.-E. S. y Solvoll, G. (2015). The importance of university facilities for student satisfaction at a Norwegian University. Facilities, 33(13/14), 744759. doi: 10.1108/F-11-2014-0081

Hoyer, R. y Hoyer, B. (2001). What is quality? Quality Progress, (Julio), 53-62. Recuperado de http://asq. org/qic/display-item/?item=14959

Hussain, K. y Birol, C. (2011). The assessment of non-academic and academic service quality in higher education. Eurasian Journal of Educa- 
tional Research, 11(42), 95-116. doi: 10.15390/ EB.2016.6187

ISO. (2015). ISO 9000_2015(en). Recuperado 22 de marzo, 2018, de https://www.iso.org/obp/ui/\#iso:std:iso:9000:ed-4:v1:en:term:3.6.2

Jiménez González, A., Terriquez Carrillo, B., y Robles Zepeda, F. J. (2011). Evaluación de la satisfacción académica de los estudiantes de la Universidad Autónoma de Nayarit. Revista Fuente, 3(6), 46-56.

Lupo, T. (2013). A fuzzy ServQual based method for reliable measurements of education quality in Italian higher education area. Expert Systems with Applications, 40(17), 7096-7110. doi: 10.1016/j. eswa.2013.06.045

Marzo Navarro, M., Pedraja Iglesias, M., y Rivera Torres, P. (2005). A new management element for universities: satisfaction with the offered courses. International Journal of Educational Management, 19(6), 505-526. doi: 10.1108/09513540510617454

Matsumoto Nishizawa, R. (2014). Desarrollo del Modelo Servqual para la medición de la calidad del servicio en la empresa de publicidad Ayuda Experto. Revista Perspectivas, (34), 181-209. Recuperado de http://www.scielo.org.bo/scielo.php?script=sci_arttext\&pid=S1994-37332014000200005\&ln$\mathrm{g}=e s \& n r m=i s o \& \operatorname{lng}=e s$

Oldfield, B. y Baron, S. (2000). Student perceptions of service quality in a UK university business and management faculty. Quality Assurance in Education, 8(2), 85-95. doi: 10.1108/09684880010325600

Parasuraman, A., Zeithaml, V. A., y Berry, L. L. (1988). SERQUAL: A Multiple-Item scale for measuring consumer perceptions of service quality. Journal of Retailing, 64(1), 12-40. doi: 10.1016/S01482963(99)00084-3

Parasuraman, A., Zeithaml, V., y Berry, L. (1985). Conceptual model of service quality and its implications for future research. Journal of Marketing, 49(4), 41 50. doi: 10.1016/S0148-2963(99)00084-3

Pereira Puga, M. (2014). Educación superior universitaria: calidad percibida y satisfacción de los egresados. Univarsidade da Coruña. Recupera- do de http://ruc.udc.es/dspace/bitstream/handle/2183/12349/PereiraPuga_Manuel_TD_2014. pdf?sequence $=2$

Rezaei, S., Karami Matin, B., Hajizadeh, M., Soroush, A., Mohammadi, Z., Babakhany, M., y Jamshidi, K. (2017). Evaluating service quality in the higher education sector in Iran: an examination of students' perspective. International Journal of Human Rights in Healthcare, 10(2), 146-155. doi: 10.1108/IJHRH-12-2016-0024

Sharif, K. y Kassim, N. M. (2012). Non-academic service quality: Comparative analysis of students and faculty as users. Journal of Marketing for Higher Education, 22(1), 35-54. doi: 10.1080/08841241.2012.705793

Sumaedi, S., Mahatma Yuda Bakti, G., y Metasari, N. (2012). An empirical study of state university students' perceived service quality. Quality Assurance in Education, 20(2), 164-183. doi: 10.1108/09684881211219424

Torres, E. y Araya, L. (2010). Construcción de una escala para medir la calidad del servicio de las universidades: Una aplicación al contexto chileno. Revista de Ciencias Sociales, 16(1), 54-67. Recuperado de http://www.scopus.com/inward/record.url?ei$d=2-s 2.0-78650531288 \&$ partnerlD=tZOtx3y1

Tumino, M. C. y Poitevin, E. R. (2013). Evaluación de la calidad de servicio universitario desde la percepción de estudiantes y docentes: caso de estudio. REICE. Revista Iberoamericana Sobre Calidad, 12(2), 63-84. Recuperado de www.rinace.net/reice/

Ur-Rashid, M.y Rhman, Z. (2017). Quality of Higher Education in Bangladesh: Application of a Modified Servqual Model. Problems of Education in the 21st Century, 75(1), 72-91. Recuperado de https://eds-b-ebscohost-com.ezp.lib.unimelb.edu.au/eds/detail/detail?vid=8\&sid=17453d2e-1979-4967-a3e3-2ff0626af3ff\%40sessionmgr103\&bdata=JnNpdGU9ZWRzLWxpd mU m c 2 NvcGU 9c $210 Z$ Q\% 3 D \% 3D\#AN $=121509807 \& \mathrm{db}=$ ehh

Recibido: 8 de setiembre de 2018 Aceptado: 20 de mayo de 2019 